\title{
Distributed Spectrum Sensing in a Cognitive Networking Testbed
}

\author{
Stefan Bouckaert ${ }^{1}$, Peter Van Wesemael $^{2}$, Jono Vanhie-Van Gerwen ${ }^{1}$, \\ Bart Jooris ${ }^{1}$, Lieven Hollevoet ${ }^{2}$, Sofie Pollin ${ }^{2}$, \\ Ingrid Moerman ${ }^{1}$, and Piet Demeester ${ }^{1}$ \\ (1) Ghent University - IBBT, \\ Gaston Crommenlaan 8 bus 201 - 9050 Ghent - Belgium. \\ (2) imec, Kapeldreef 75 - 3001 Leuven - Belgium.
}

\begin{abstract}
In this demonstration, we show how the IBBT w-iLab.t wireless testbed, combined with multiple spectrum sensing engines designed by imec, can be used for experimentally-supported design and evaluation of cognitive networking protocols. Functionalities include the advanced characterization of the behavior of a cognitive solution under test, and characterization of the wireless experimentation environment itself.
\end{abstract}

Keywords: experimentation, testbed, wireless, cognitive radio, cognitive networking, spectrum sensing

\section{Introduction}

The field of experimentally supported research in wireless networks receives increasing attention from the international research community. Multiple wellestablished wireless networks such as TWIST, w-iLab.t, Motelab, or Orbit have now been in use for multiple years, and many of these testbeds are now also putting effort in federating their facilities. One of these federations, the CREW (Cognitive Radio Experimentation World) federation (www.crew-project.eu), is bringing together the hardware, software, and expertise from multiple European wireless testbeds, with the aim of facilitating experimental research in the field of cognitive radio, cognitive networking and advanced spectrum sensing.

\section{System Description and Novel Characteristics}

The presented demonstration is an evolution of our contribution at the ServiceWave 2010 conference, entitled "Spectrum Sharing in Heterogeneous Wireless Networks: An FP7 CREW Use Case". In last year's demonstration, we showcased both a cognitive networking experiment that was remotely deployed on the IBBT w-iLab.t (a heterogeneous ISM testbed with $200 \mathrm{Wi}-\mathrm{Fi}$ and Zigbee based wireless nodes deployed over three floors of an office environment), and the realtime use of the imec sensing engine in the exhibition area. Since then, the imec sensing engine has considerably progressed: while processing of the received wireless signal used to be done in Matlab, it is now running in real-time on a newly 
developed ASIP, called the DIFFS (Digital Interface for Sensing). The DIFFS enables flexible processing through a wide variety of sensing algorithms, including cyclostationary detection for DVB-T, resource allocation detection for LTE, and energy detection. As a result, we will now be able to showcase a portable version of the sensing solution, equipped with a USB interface.

Furthermore, we have now integrated this new version of the spectrum sensing engine in the w-iLab.t testbed: currently, the sensing engine is configured to scan the entire $2.4 \mathrm{GHz}$ ISM band, and continuously puts power spectral density (PSD) values in a database. This information is then visualized in real-time on the w-iLab.t GUI and may also be used during or after the experiment by the experimenter. Soon (during the demo), ten configurable spectrum sensing engines will be installed permanently throughout the w-iLab.t testbed.

In addition to showcasing the new sensing hardware and the integration, we demonstrate the use of these multiple distributed sensing engines inside the w-iLab.t environment, in an example cognitive networking use case where a Zigbee-based wireless sensor network reconfigures its transmission parameters in order to better coexist with a co-located Wi-Fi network.

The availability of distributed sensing components in a wireless testbed, brings many new possibilities, including (i) defining metrics based on the acquired PSD values when evaluating wireless (cognitive) protocols; (ii) capturing the state of the wireless environment in the testbed, before, after and during an experiment, thus increasing the meaningfulness and comparability of experimental results; (iii) developing novel cognitive networking protocols, which are using the detailed distributed spectral measurements to optimize their transmission parameters. We will showcase the above use case by setting up a repeatable, emulated home environment: first, a set of devices in the w-iLab.t testbed is configured as access points and clients; second, the reliability of a simple, nonoptimized, sensor network solution is characterized in terms of packet loss and spectral efficiency. Third, the experiment will be repeated, but now with the sensor network performing cognitive channel selection. Again, the reliability and spectral efficiency of the solution will be evaluated.

To summarize, this demonstration complements and extends last year's demonstration, by presenting the completed integration of our cognitive networking testbed and our updated, more compact and faster spectrum sensing engine. Moreover, we demonstrate how this combination enables us to emulate a repeatable house environment and how our example coexistence experiment can be monitored and evaluated in real-time.

A short demonstration video can be found at: http://users.atlantis.ugent.be/sbouckae/hidden/crew.

\section{Acknowledgement}

The research leading to these results has received funding from the European Union's Seventh Framework Programme (FP7/2007-2013) under grant agreement n 258301 (CREW project). 